\title{
Control de tráfico vehicular usando ANFIS
}

\author{
Vehicular traffic control using ANFIS \\ Luis Fernando Pedraza ${ }^{1} \quad$ César Augusto Hernández ${ }^{1} \quad$ Danilo Alfonso López ${ }^{1}$ \\ Recibido 10 de marzo de 2011, aceptado 15 de marzo de 2012 \\ Received: March 10, 2011 Accepted: March 15, 2012
}

\begin{abstract}
RESUMEN
Diferentes estrategias para el control del tráfico urbano se han presentado a lo largo del tiempo. Este artículo presenta el diseño de un modelo de tráfico vehicular, el cual examina el tráfico existente en una vía a través de una serie de semáforos. A partir de este modelo se sincronizan los tiempos de duración y de desfase de los semáforos, utilizando para ello el Sistema de Inferencia Difusa Basado en Redes Adaptativas (ANFIS). El modelo es simulado y los resultados se evalúan a nivel macroscópico con el modelo de tiempos fijos, que funciona actualmente en Bogotá-Colombia.
\end{abstract}

Palabras clave: Parámetros macroscópicos, tiempos fijos, tráfico vehicular, redes adaptativas, lógica difusa, suma del error al cuadrado.

\begin{abstract}
Different strategies for urban traffic control have been presented over time. This paper presents the design of a vehicular traffic model, examining the existing traffic through a serie of traffic lights on a road. From this model the times of duration and phase of the traffic lights are synchronized, using the Adaptive Network Based Fuzzy Inference Systems (ANFIS). The model is simulated and the results are evaluated at macroscopic level with the fixed time model, currently operating in Bogota-Colombia.
\end{abstract}

Keywords: Macroscopic parameters, fixed time, vehicle traffic, adaptive networks, fuzzy logic, sum of squared error.

\section{INTRODUCCIÓN}

A lo largo de la historia de las grandes capitales del mundo, el flujo vehicular se ha incrementado constantemente, no obstante, el desarrollo de la infraestructura vial ha sido insuficiente; esto se ha reflejado en situaciones donde el volumen de tránsito en uno o más puntos de una vía excede el volumen máximo que puede pasar por ellos. Por lo tanto, el control de tráfico inteligente es una herramienta muy importante para el control de la congestión vehicular y beneficiar así la calidad de vida de las personas, el medio ambiente y la economía de la ciudad.
Por ejemplo, el transporte particular que se desplazó por la red vial de Bogotá para el año 2010 constó de aproximadamente 1.063 .869 automóviles particulares matriculados [1], los cuales manejan solo un $22 \%$ de los viajes diarios en la ciudad. Durante el 2010 en Bogotá, transitaron 17.083 vehículos de transporte público, entre los que se encuentran buses, busetas, microbuses, transmilenios (sistema de transporte masivo) y 49.206 taxis. Estos vehículos realizaron el $56 \%$ de los viajes en la ciudad y el restante $22 \%$ estuvo cubierto por desplazamientos a pie, motocicletas y bicicletas, entre otros [1]. Si el parque automotor crece a una tasa del $4 \%$ anual (como en los últimos años), se calcula que para el 2015 Bogotá tendría aproximadamente 1.212.000 vehículos, y si

\footnotetext{
1 Universidad Distrital Francisco José de Caldas. Cr 7 No 40-53. Bogotá, Colombia. E-mail: lfpedrazam@udistrital.edu.co; cahernadezs@udistrital.edu.co; dalopezs@udistrital.edu.co
} 
crece al $11 \%$ como en los años 2009 y 2010 habría 1.800 .000 vehículos. Este crecimiento inminente ha llevado y llevará a grandes congestiones vehiculares, que hoy por hoy son uno de los problemas más grandes de la capital colombiana, trayendo como consecuencia la necesidad de un óptimo sistema de control que permita un ágil desplazamiento y que reduzca entre otros el alto consumo de combustibles, la alta contaminación ambiental causada por el desprendimiento de monóxidos propio de los automotores y el nivel de accidentalidad.

En los últimos años el paradigma de control del flujo vehicular mediante la programación de los semáforos ha presentado múltiples variaciones que van desde la programación con tiempos fijos en las diferentes fases de los semáforos hasta la coordinación de grupos desde una central computarizada, lo que demuestra que se han obtenido grandes avances en el tema. Por ejemplo, en [2] se hace un estudio de factor de utilización de los carriles de algunas intersecciones semaforizadas de Bogotá, teniendo en cuenta los tiempos de operación actual de los semáforos de estas intersecciones, en [3] se hace una revisión de las estrategias de control de los sistemas de tráfico más utilizadas en Colombia, en [4] se describe un controlador de lógica difusa para una sola intersección, en [5] se estudia el uso de la lógica difusa para el control de intersecciones múltiples, en [6] se describe una red de caminos conectados por semáforos basados en sistemas expertos, en [7] se presenta un controlador de semáforos usando un predictor simple basado en optimización, en [8] se desarrolla un controlador de tráfico vehicular basado en algoritmos evolutivos, en [9] se presenta un método de control de semáforos basado en aprendizaje reforzado, el cual en [10] utiliza un valor basado en la función del semáforo a partir de una red neuronal y en [11] se usa la función basada en vehículos. En [12] se presenta un modelo neurodifuso para el control de semáforos. De tal forma, que existen diferentes propuestas al problema de tráfico vehicular, pero a su vez existe un gran campo de trabajo, como lo es el modelo presentado en este artículo para optimizar el tiempo de sincronismo de los semáforos.

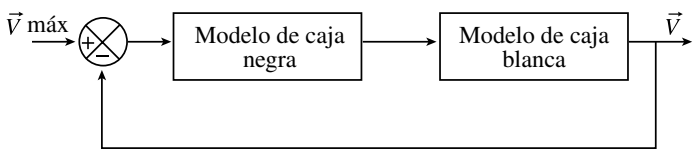

Figura 1. Estructura del sistema.
Por lo tanto, la intención es proporcionar un enfoque en el manejo de la semaforización en Bogotá de acuerdo a los requerimientos de tránsito en una vía, basado en técnicas de control inteligente como lo es ANFIS, el cual ha demostrado una gran adaptabilidad y capacidad para toma de decisiones en sistemas con fluctuaciones como lo es el tráfico [13]. El objetivo es controlar un modelo de caja blanca (expresión matemática) con un modelo de caja negra (ANFIS), como se observa en la Figura 1.

\section{DISEÑO DEL CONTROLADOR DE TRÁFICO}

Inicialmente se presenta un modelo de tráfico macroscópico, el cual es el punto de partida para diseñar el control de los tiempos de verde de los semáforos y los respectivos desfases entre estos utilizando el ANFIS.

\section{Descripción del modelo}

En [14] se introduce un modelo microscópico de tráfico vehicular; prácticamente es un modelo ideal basado en el comportamiento cinemático de un solo vehículo que viaja a través de una secuencia de semáforos que encienden y apagan con una frecuencia específica y que presentan distancias fijas (para este caso $100 \mathrm{~m}$ ).

Partiendo de esta investigación, el primer objetivo es analizar una corriente vehicular (varios vehículos) a través de una secuencia de semáforos que pueda presentar distancias variables entre ellos y luego controlar estos semáforos para pretender mantener la velocidad máxima de la corriente vehicular permitida en la vía.

A continuación se describen los parámetros que componen la función:

La separación entre el $n$-ésimo y el $(n+1)$-ésimo semáforo es $L n$. La n-ésima luz es verde si sen ( $\omega n t$ $+\varphi n)>0$ y roja en caso contrario, donde $\omega n$ es la frecuencia del semáforo y $\varphi n$ es el desfase entre semáforos. Un automóvil en esta secuencia de semáforos puede tener:

- Una aceleración positiva $a_{+}$hasta que su velocidad alcanza la velocidad máxima de la vía (vmáx). 
- Una velocidad constante vmáx con aceleración nula.

- Una aceleración negativa $-a_{-}$hasta que se detiene.

$$
\frac{d v}{d t}=\left\{\begin{array}{lc}
a_{+} \theta\left(v_{\text {máx }}-v\right), & \text { acelera } \\
-a_{-} \theta(v), & \text { frena }
\end{array}\right.
$$

En la ecuación (1), $\theta(v)$ es la función escalón unitario, la cual impide que el modelo arroje velocidades superiores a la máxima e inferiores a cero.

Como el auto se acerca al n-ésimo semáforo con velocidad $v$ el conductor debe tomar una decisión, frenar o no en la distancia $X c$ :

$$
X c=\frac{V^{2}}{2 a_{-}}
$$

La diferencia entre $L n$ y $X c(L n-X c)$, es la distancia a la cual el vehículo puede acelerar hasta alcanzar la vmáx.

Para el modelo macroscópico en el que se maneja un flujo vehicular, cada vehículo acelera con una aceleración $a_{+}$, se supone que el primer vehículo en la fila posee una $a_{+}=2,32 \mathrm{~m} / \mathrm{s}^{2}$ y que cada vehículo que lo precede disminuye su $a_{+}$en un $2 \%$, y se evalúa en cada $\Delta t$ la diferencia entre $X$ y $X c$ ( $X$ es la distancia recorrida por el vehículo), cuando esta sea menor a $1 \mathrm{~m}$ se analiza el estado del semáforo, es decir, $\operatorname{sen}(\omega n t+\varphi n)$, si el resultado es positivo se continúa con $a_{+}$y por, el contrario, se actualiza el valor de aceleración a $a_{-}$, que en este caso es de $-3,9 \mathrm{~m} / \mathrm{s}^{2}$. De esta forma se calculan los datos de velocidad instantánea con los cuales se obtienen los datos de la velocidad promedio para los $m$ autos del flujo vehicular.

La velocidad $v$ de la ecuación (1) se reemplaza por el promedio de la velocidad promedio de los $m$ vehículos $(V t)$, es decir:

$$
\bar{V}_{t}=\frac{\sum_{i=1}^{m} V_{i}}{m}
$$

En la ecuación (3) $V_{i}$ es la velocidad promedio del m-ésimo vehículo. Esta velocidad promedio se obtiene a partir de la velocidad instantánea del vehículo $\left(V_{i n}\right)$, la cual se calcula cada 0.1 segundos $(\Delta t)$, mientras el vehículo se desplaza en el flujo vehicular del semáforo $n$ hasta el $n+1$. Donde:

$$
V_{\text {in }}=V_{0}+a_{+} \Delta t
$$

En la ecuación (4) $V_{0}$ es la velocidad que lleva el vehículo en el intervalo de tiempo.

Luego de describir el modelo a utilizar para caracterizar el flujo de vehículos a través de una vía, se utiliza la ecuación (5) para sincronizar los tiempos de verde $\left(t_{v}\right)$ y de desfase $\left(t_{d}\right)$ de los semáforos con el modelo ANFIS:

$$
\operatorname{sen}\left(\frac{2 \pi}{t_{V n}+t_{A n}+t_{R n}+t_{R A n}} t+t_{d n} \omega_{n}\right)>0
$$

En la ecuación (5),

$t_{v n}$ es el tiempo de verde del $n$-ésimo semáforo.

$t_{A n}$ es el tiempo de amarillo del $n$-ésimo semáforo. $t_{R n}$ es el tiempo de rojo del $n$-ésimo semáforo.

$t_{R A n}$ es el tiempo de rojo-amarillo del $n$-ésimo semáforo.

$t_{d n}$ es el tiempo de desfase de la luz verde entre el n-ésimo semáforo y el (n-1)-ésimo semáforo.

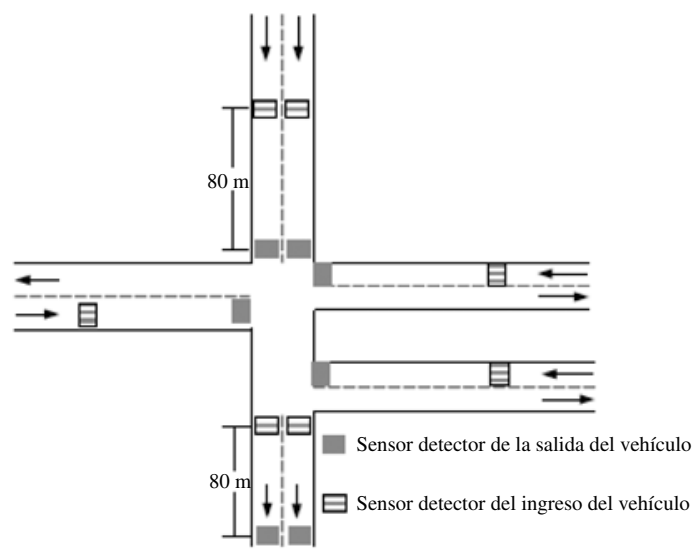

Figura 2. Ubicación de los sensores en la avenida.

Adicionalmente se deben incluir las restricciones consideradas en este modelo, las cuales consisten en tener un número máximo de 20 vehículos en un carril partiendo del reposo; se ubican sensores de bucle magnético a 80 metros de distancia con respecto al semáforo y a ambos lados de la vía para lograr una detección de un número máximo de 
aproximadamente 40 vehículos, como se muestra en la Figura 2. Para esto se tiene en cuenta que la longitud promedio del vehículo es de $4 \mathrm{~m}$ y la separación entre vehículos se encuentra a partir del modelo lineal planteado en [15], [16], [17]. Partiendo de la información dada, en [18] en donde el volumen máximo vehicular $\left(q_{m}\right)$ que se presenta sobre la avenida Caracas es de alrededor de 2.736 vehículos/hora y como la velocidad máxima (vmáx) permitida en la vía es de $60 \mathrm{~km} / \mathrm{h}(16,67 \mathrm{~m} / \mathrm{s})$, se tiene que la densidad de congestionamiento vehicular $\left(k_{c}\right)$ es:

$$
k_{c}=\frac{4 * q_{m}}{v m a ́ x}=182,4 v e h / \mathrm{km}
$$

El espaciamiento promedio a flujo máximo $\left(s_{m}\right)$ es:

$$
s_{m}=\frac{1}{k_{m}}=\frac{1}{\frac{k_{c}}{2}}=10,96 \mathrm{~m} / \mathrm{veh}
$$

Y la separación entre vehículos a flujo máximo $\left(s_{p}\right)$ es:

$$
s_{p}=s_{m}-l_{v}=6,96 m / v e h
$$

Donde $l_{v}$ es la longitud promedio del vehículo [15], [19].

\section{Diseño del controlador}

Ahora se procede a diseñar el controlador ANFIS para asignar el tiempo de verde de los semáforos y sus respectivos desfases, buscando obtener la velocidad máxima permitida en la vía. Este diseño se realiza para controlar el modelo descrito en el ítem anterior.

Para el entrenamiento del modelo neurodifuso, las reglas difusas del tipo Takagi-Sugeno se representan de la siguiente forma [20]:

Regla n: Si $X$ es $A_{n}$ y $Y$ es $B_{n}$, entonces $f_{n}=p_{n} X+$ $q_{n} Y+r_{n}$, en el caso de dos semáforos la arquitectura de las reglas implementadas es:

Regla 1: Si $X$ es $A_{l}$ y $Y$ es $B_{l}$, entonces $f_{l}=p_{l} X$ $+q_{1} Y+r_{1}$

Regla 2: Si $X$ es $A_{2}$ y $Y$ es $B_{2}$, entonces $f_{2}=p_{2} X$ $+q_{2} Y+r_{2}$
Donde $A_{n}$ y $B_{n}$ son los conjuntos difusos de entrada y $p_{n}, q_{n}$ y $r_{n}$ son constantes, para este caso $p_{n}$ y $q_{n}$ son cero obteniendo un modelo Takagi-Sugeno de orden cero. Los niveles de activación de las reglas se calculan como:

$$
w_{n}=A_{n}(x) \cdot B_{n}(y), \mathrm{n}=1,2 \ldots
$$

En la ecuación (9) el operador lógico (.) es modelado por una t-norma continua (producto). Las salidas individuales de cada regla son obtenidas como una combinación lineal entre los parámetros del antecedente de cada regla y la salida de control del modelo $f_{n}$ se obtiene por la normalización de los grados de activación de las reglas por la salida individual de cada regla:

$$
\begin{gathered}
f=\frac{w_{1} f_{1}+w_{2} f_{2}}{w_{1}+w_{2}} \\
f=\bar{w}_{1} f_{1}+\bar{w}_{2} f_{2}
\end{gathered}
$$

En la ecuación (10b) $\overline{\mathrm{w}}_{1}$ y $\overline{\mathrm{w}}_{2}$ son los valores normalizados de $w_{1}$ y $w_{2}$ con respecto a la suma $\left(w_{1}\right.$ $+w_{2}$ ). La red neuronal híbrida que representa este tipo de inferencia es una red adaptativa con cinco capas, donde cada capa representa una operación del mecanismo de inferencia difuso. Esta red se muestra en la Figura 3.

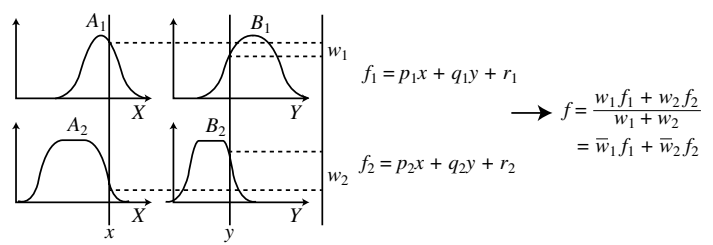

a)

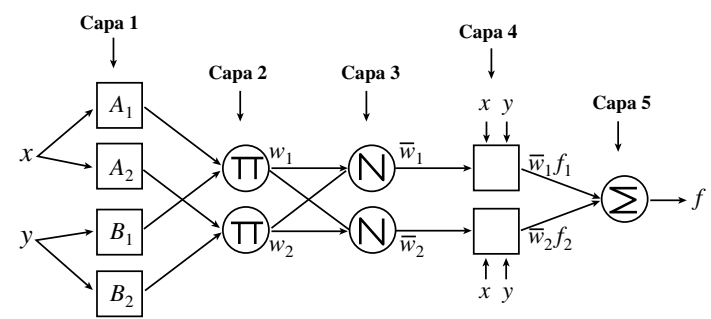

b)

Figura 3. Arquitectura ANFIS para dos semáforos. a) Relaciones difusas. b) Equivalente ANFIS. 
$X$ es el antecedente (universo de entrada) tiempo de verde $\left(t_{v}\right), Y$ es el antecedente (universo de entrada) tiempo de desfase $\left(t_{d}\right)$ de la ecuación (5), y $f$ corresponde al consecuente (universo de salida) velocidad promedio del flujo vehicular $\left(V_{t}\right)$, dada en la ecuación (3). El modelo implementado en el software Matlab es mostrado en la Figura 4.

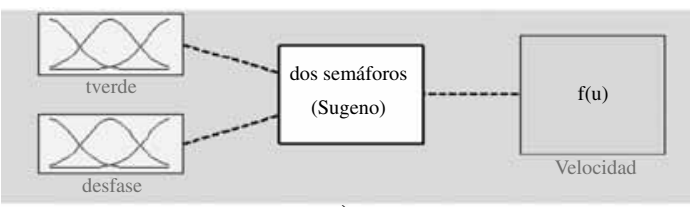

a)

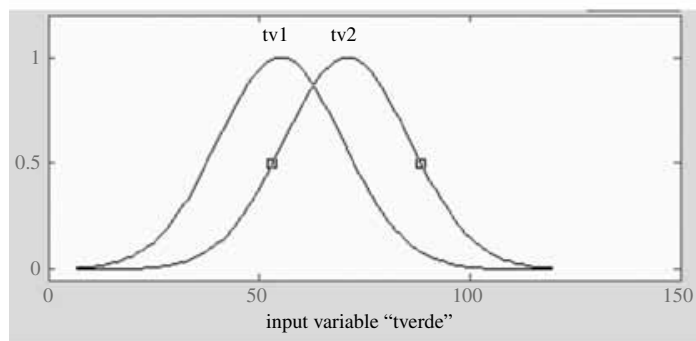

b)

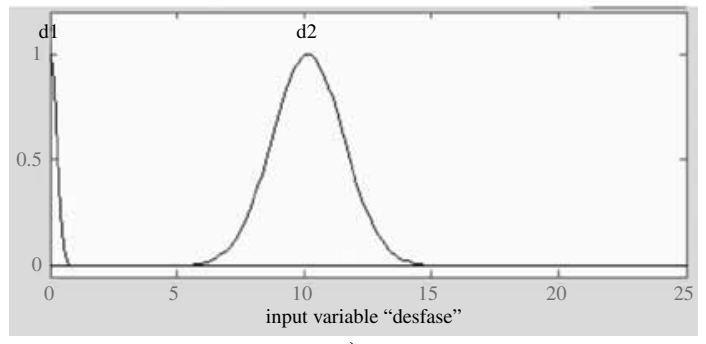

c)

Figura 4. Universos para el sistema ANFIS. a) Universos ANFIS basados en el modelo Takagi-Sugeno. b) Conjuntos gaussianos del universo $t_{v}$. c) Conjuntos gaussianos del universo desfase.

En este caso se utilizan conjuntos de entrada gaussianos, los cuales están definidos por:

$$
\mu_{A i}(x)=\exp \left(-\left(\frac{x-c_{i}}{a_{i}}\right)^{2}\right)
$$

El parámetro $C_{i}$ es el valor medio del conjunto, y $a_{i}$ es el coeficiente de dispersión. En este caso $C_{i}$ indica el tiempo de verde y el desfase necesarios para ajustar el modelo, como se muestra en la
Figura 4 b) y c). A continuación se muestra la forma en que se entrenan los parámetros para asignar el tiempo de verde y el desfase con el fin de alcanzar la velocidad máxima.

\section{Aprendizaje del modelo}

El modelo ANFIS tiene dos tipos de parámetros que deben ser entrenados:

1. Los parámetros de los antecedentes $\left(t_{v}, \mathrm{y} t_{d}\right)$, para lo cual se encuentran las constantes que caracterizan los conjuntos gaussianos para este caso el $t_{v n}$ y el $t_{d n}$, son el resultado del centro del conjunto.

2. Los parámetros del consecuente $\left(V_{T}\right)$ son parámetros lineales de la salida del modelo de inferencia.

El paradigma de aprendizaje del modelo ANFIS emplea algoritmos de gradiente descendente para optimizar los parámetros del $t_{v}$ y el $t_{d}$ y el algoritmo de mínimos cuadrados para determinar los parámetros lineales del $V_{T}$. A esta combinación se la conoce como regla de aprendizaje híbrido, la cual es usada en el aprendizaje de este modelo [21].

Ahora se aplica lo descrito en [21] y [22], en donde para aplicar el aprendizaje híbrido en grupo, en cada periodo de entrenamiento debe ejecutarse un paso hacia adelante y un paso hacia atrás. En el paso hacia adelante, los parámetros de las funciones de los conjuntos son inicializados y se presenta un vector de entrada-salida, se calculan las salidas del nodo para cada capa de la red y entonces los parámetros de la $V_{T}$ son calculados usando el método de mínimos cuadrados. Una vez identificados los parámetros de la $V_{T}$, el error es calculado como la diferencia entre la salida de la red y la salida deseada presentada en las parejas de entrenamiento. En este caso se usa una de las medidas más empleadas para calcular el error de entrenamiento, la cual es la suma del error al cuadrado (SEC), definido como:

$$
S E C=\sum_{k=1}^{N}\left(d_{k}-f_{k}\right)^{2}
$$

En la ecuación (12) $d_{k}$ corresponde a los patrones de entrenamiento proporcionados (salidas deseadas) y $f_{k}$ es la correspondiente salida de la red. En el paso hacia atrás, las señales de error son propagadas 
desde la salida, en dirección de las entradas y el vector gradiente es acumulado para cada dato de entrenamiento. Al final del paso hacia atrás para todos los datos de entrenamiento, los parámetros en la capa 1 (parámetros de los conjuntos) son actualizados por el método descendente en una magnitud $\Delta \alpha$ igual a:

$$
\Delta \alpha=-\eta \frac{\partial E}{\partial \alpha}
$$

Donde en la ecuación (13) $E$ es el error de salida y $\eta$ es la velocidad de aprendizaje que puede ser expresada como:

$$
\eta=\frac{\rho}{\sqrt{\sum_{\alpha}\left(\frac{\partial E}{\partial \alpha}\right)^{2}}}
$$

En la ecuación (14) $\rho$ es el tamaño del paso, o sea la longitud de cada transición a lo largo de la dirección del gradiente en el espacio de parámetros. Generalmente, se puede cambiar el valor de $\rho$ para variar la velocidad de convergencia de la siguiente manera:

Se observa que si $\rho$ es pequeño, el método del gradiente aproxima de cerca la trayectoria del gradiente, pero la convergencia será lenta puesto que el gradiente se debe calcular muchas veces. Por otra parte, si $\rho$ es grande, la convergencia será inicialmente muy rápida, pero el algoritmo oscilará sobre el grado óptimo. De acuerdo con estas observaciones, se actualiza $\rho$ según las siguientes reglas heurísticas:
1. Si la medida del error experimenta cuatro reducciones consecutivas, se aumenta $\rho$ en el $10 \%$.

2. Si la medida del error experimenta dos combinaciones consecutivas de un aumento y de una reducción, se disminuye $\rho$ en un $10 \%$.

Sin embargo, los valores de cuatro reducciones, dos combinaciones y el aumento o disminución de $\rho$ en el $10 \%$, son elegidos de manera un poco arbitraria, aunque, como se observa más adelante, muestran un buen resultado en la respuesta de la $V_{T}$, lo importante es no escoger un valor inicial de $\rho$ muy grande, para que este no sea crítico [23].

\section{VALIDACIÓN DEL MODELO}

Para la validación del modelo se utiliza la Avenida Caracas de Bogotá D.C., desde el cruce de la Calle 36 hasta el cruce de la Calle 51 (en total ocho cruces) en sentido sur a norte, para lo cual se valida el modelo propuesto confrontándolo con el de tiempos fijos coordinado por la Secretaría de Movilidad de Bogotá. Las características actuales de estos cruces, como lo son distancia entre cruces y tiempos de semáforos, se presentan en la Tabla 1, donde se observa que los primeros cuatro cruces tienen un tiempo promedio de ciclo (tiempo de rojo más tiempo de verde) de 96 segundos y para los cuatro cruces siguientes este tiempo es de aproximadamente 115 segundos. Los tiempos de Rojo-Amarillo y Amarillo para los semáforos de los cruces son de un segundo y dos segundos respectivamente.

Las gráficas de velocidad promedio de los vehículos, a través de los ochos semáforos para el modelo de

Tabla 1. Datos de ocho cruces de la Av. Caracas.

\begin{tabular}{|c|c|c|c|c|}
\hline $\begin{array}{c}\text { Cruce } \\
\text { Sur a Norte }\end{array}$ & $\begin{array}{c}\text { Longitud entre } \\
\text { cruces (m) }\end{array}$ & Tiempo de rojo(s) & $\begin{array}{c}\text { Tiempo de } \\
\text { verde(s) }\end{array}$ & $\begin{array}{c}\text { Desfase temporal } \\
\text { de verde (s) }\end{array}$ \\
\hline Av. Carac. No 36 & - & 41 & 54 & - \\
\hline Av. Carac. No 37 & 143 & 33 & 65 & 0 \\
\hline Av. Carac. No 39 & 256 & 45 & 51 & 13 \\
\hline Av. Carac. No 42 & 208 & 37 & 56 & 6.7 \\
\hline Av. Carac. No 45 & 281 & 49 & 66 & - \\
\hline Av. Carac. No 47 & 212 & 47 & 68 & 4.5 \\
\hline Av. Carac. No 49 & 226 & 49 & 68 & 0.5 \\
\hline Av. Carac. No 51 & 224 & 43.5 & 72 & 2.2 \\
\hline
\end{tabular}


tiempos fijos y el modelo propuesto, se realizan para una cantidad de 40 vehículos. En la Figura 5 se grafica el modelo de tiempos fijos para los tiempos y distancias dados en la Tabla 1, allí se ve que el sistema presenta un comportamiento caótico para un volumen apreciable de vehículos, esto conlleva a que la velocidad promedio sea de $6,9 \mathrm{~m} / \mathrm{s}$ $(24,83 \mathrm{~km} / \mathrm{h})$.

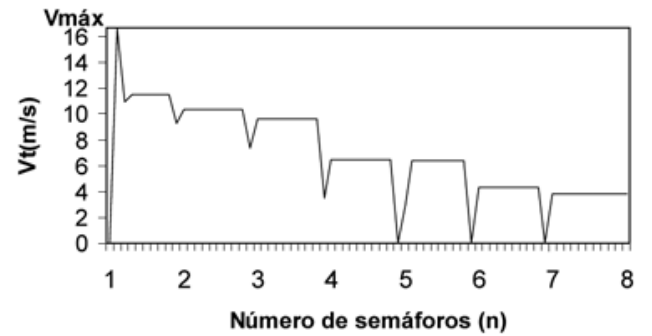

Figura 5. Velocidad promedio de 40 vehículos a través de ocho semáforos de tiempos fijos.

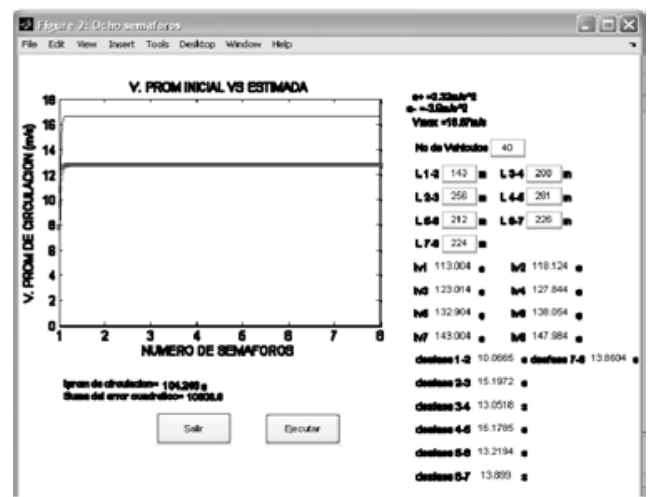

Figura 6. Velocidad promedio de 40 vehículos a través de ocho semáforos sincronizados con el modelo ANFIS.

En la Figura 6 se observan los tiempos de verde $\left(t v_{n}\right)$ y el desfase entre cada uno de los semáforos devueltos por el modelo ANFIS y además se muestra en color claro la velocidad promedio del flujo vehicular que se desea alcanzar (en este caso 16,67 m/s) y en color oscuro la velocidad promedio que realmente se alcanza con los tiempos y desfases de los semáforos proporcionados por el modelo, aquí la velocidad promedio es de $12,77 \mathrm{~m} / \mathrm{s}$. A su vez se muestra el tiempo promedio de circulación de los vehículos, el cual es aproximadamente 104 segundos y la suma del error al cuadrado es de 10.606, dada por el error de entrenamiento de la red neuronal a través de
10 periodos. Vale la pena aclarar que en este caso el ciclo total de cada semáforo es de 3 minutos, de los cuales el máximo tiempo que puede ser destinado para verde es de 149 segundos, el mínimo tiempo de rojo es de 28 segundos, el tiempo de rojo-amarillo es de 1 segundo y el de amarillo es de 2 segundos.

\section{RESULTADOS}

Ahora se procede a realizar una comparación con los parámetros macroscópicos de los dos modelos (ANFIS y tiempos fijos), para esto se parte de un volumen vehicular máximo de 2.736 vehículos/ hora [18]. A partir de este volumen, se realiza su tabulación, en periodos de 20 minutos durante una hora, como se muestra en la Figura 7 a). Esto es realizado a través de los ocho semáforos de la Av. Caracas descritos en la Tabla 1, los cuales actualmente se encuentran con tiempos fijos y estos son comparados con los tiempos arrojados por el modelo ANFIS diseñado en el capítulo anterior. En la Figura 7 b) se observa la densidad vehicular, en la c) la velocidad promedio máxima alcanzada por los vehículos y en la d) la longitud promedio de vehículos en cola sobre las ocho colas, para cada uno de los modelos en discusión.

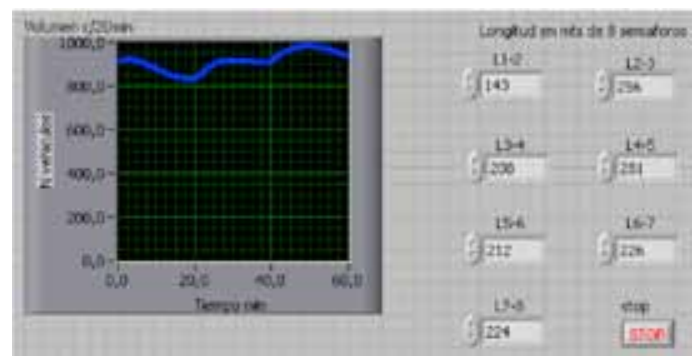

a)

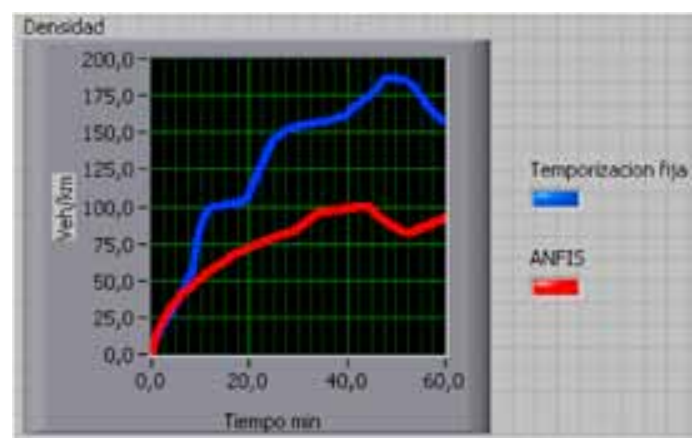

b) 


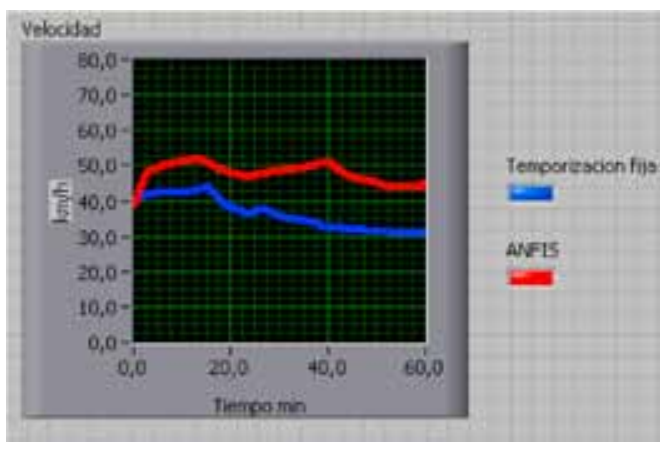

c)

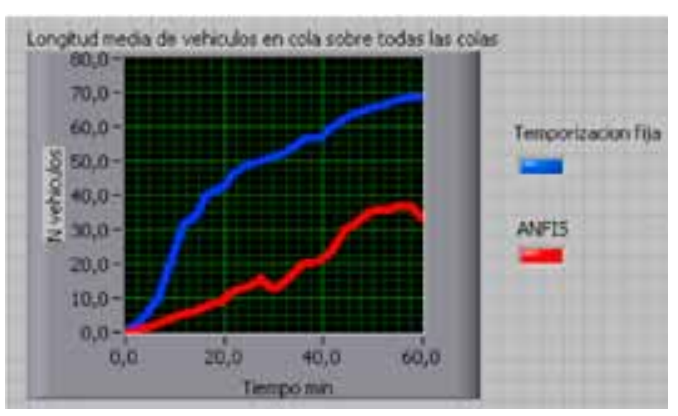

d)

Figura 7. Comparación macroscópica del modelo ANFIS con el modelo de tiempos fijos. a) Volumen vehicular para periodos de 20 minutos a través de una hora. b) Densidad vehicular en veh $/ \mathrm{km}$. c) Velocidad promedio vehicular en $\mathrm{km} / \mathrm{h}$. d) Número promedio de vehículos en cola sobre todas las colas.

\section{Análisis de resultados}

Al comparar la velocidad promedio de los 40 vehículos en los dos modelos, como se muestra en las Figuras 5 y 6 , puede observarse un comportamiento estable para el modelo ANFIS y una velocidad promedio mayor, aproximadamente un $85 \%$ con respecto al modelo de tiempos fijos.

El controlador diseñado con ANFIS gestiona de una mejor manera el tráfico vehicular que el sistema de tiempos fijos como se describe en el siguiente análisis: En la Figura 7 b) el promedio de densidad vehicular se reduce de 136 vehículos $/ \mathrm{km}$ a 78 vehículos $/ \mathrm{km}$, aproximadamente en un $42,6 \%$ con respecto al sistema de tiempos fijos, en la Figura 7 c) la velocidad promedio para el modelo ANFIS es de $48,1 \mathrm{~km} / \mathrm{h}$ mientras que para el de tiempos fijos es de $36 \mathrm{~km} / \mathrm{h}$, en este caso la velocidad aumenta en un $33,3 \%$ con el modelo propuesto y en la Figura 7 d) la cantidad promedio de vehículos en cola sobre todas las colas para el sistema de tiempos fijos es de 48,75 vehículos y para el modelo ANFIS es de 18,65 vehículos, o sea se tiene una reducción con el modelo ANFIS en la cantidad de vehículos en cola de un $61,7 \%$.

\section{CONCLUSIONES}

$\mathrm{Al}$ analizar los resultados de la Figura 7 se observa que el control de los semáforos con el modelo ANFIS es más óptimo, ya que la densidad vehicular se reduce, permitiendo atender una mayor cantidad de vehículos en una misma distancia al compararse con el sistema de tiempos fijos. A su vez el modelo ANFIS aumenta la velocidad promedio de los vehículos a través de la vía, lo cual aumenta la movilidad vehicular, y también se presenta una disminución del promedio de vehículos en cola sobre todas las colas, reduciendo la posibilidad de generar una congestión vehicular por causa de los semáforos.

Para la obtención de buenos resultados con este modelo es aconsejable utilizarlo en un sistema progresivo flexible, el cual permita sincronizar los semáforos en un sentido u otro, según las horas de máxima demanda vehicular.

La red adaptativa del modelo ANFIS se entrena durante 10 periodos, con lo que se alcanza un tiempo corto de ejecución. A medida que los periodos de entrenamiento de la red se aumentaron no se observó una mejora significativa en la velocidad de circulación vehicular y en cambio sí un aumento significativo en el tiempo de ejecución.

La implementación del modelo propuesto en Bogotá conllevaría a unos beneficios de índole social entre los que se encuentran:

- El aumento de la calidad de vida de los ciudadanos, puesto que permite a las personas que se transportan en automotores llegar a su destino sin mayores contratiempos y ocupar así el tiempo en otras labores como su familia.

- Disminución del nivel de ruido, ya que los conductores accionarían la bocina con menor frecuencia. A su vez el vehículo disminuiría la expulsión de gases, lo cual representa no solo 
una reducción en la contaminación ambiental, sino en el consumo de combustible, lo que implica un costo inferior en el sostenimiento del vehículo para el dueño.

Como trabajo futuro se recomienda realizar el modelo teniendo en cuenta que si un volumen grande de vehículos congestiona un cruce anterior, el número de vehículos que viene de ese cruce sea reducido. También es recomendable incluir en el modelo factores que afectan al conductor como lo es el clima, la época del año, entre otros.

\section{REFERENCIAS}

[1] Cámara de Comercio de Bogotá. "Observatorio de movilidad". Septiembre 2011. ISSN 2027-209. URL: http://www.ccb.org.co

[2] R. Peña. "Análisis de los factores de ajuste por utilización de carril en intersecciones semaforizadas de Bogotá D.C.'. Ingeniería e Investigación. Vol. 30, No 1, pp. 5-10. 2010.

[3] D. Robles, P. Ñañez y N. Quijano. "Control y simulación de tráfico urbano en Colombia: Estado del arte". Revista de Ingeniería Universidad de los Andes. No 29, pp. 59-69. 2009.

[4] K.K. Tan, M. Khalid and R. Yusof. "Intelligent traffic lights control by fuzzy logic". Malaysian Journal of Computer Science. Vol. 9, Issue 2, pp. 29-35. December, 1996.

[5] J.H. Lee, K.M. Lee, K.A. Seong, C.B. Kim and H. Lee-Kwang. "Traffic control of intersection group based on fuzzy logic". Proceedings of the 6th International Fuzzy Systems Association World Congress, pp. 465-468. 1995.

[6] N. Findler and J. Stapp. "A distributed approach to optimized control of street traffic signals". Journal of Transportation Engineering. Vol. 118-1, pp. 99-110. 1992.

[7] K. Tavladakis and N.C. Voulgaris. "Development of an autonomous adaptive traffic control system". The European Symposium on Intelligent Techniques. June, 1999.

[8] H. Taale, Th. Back, M. Preuß, AE. Eiben, J.M. de Graaf and C.A. Schippers. "Optimizing traffic light controllers by means of evolutionary algorithms". The European
Congress on Intelligent Techniques and Soft Computing. Vol. 3, pp. 1730-1734. 1998.

[9] T.L. Thorpe and C. Andersson. "Traffic light control using sarsa with three state representations". Technical Report, IBM corporation. 1996.

[10] T. Thorpe. "Vehicle traffic light control using sarsa". Master tesis. Department of Computer Science, Colorado State University. Fort Collins. USA. 1997.

[11] M. Wiering, J. Vreeken, J. Van Veenen and A. Koopman. "Simulation and optimization of traffic in a city". Intelligent Vehicles Symposium, pp. 453-458. June, 2004.

[12] O. Salcedo, L. Pedraza y C. Hernández. "Modelo de Semaforización Inteligente para la Ciudad de Bogotá". Revista de Ingeniería Universidad Distrital. Vol. 11, $\mathrm{N}^{\circ}$ 2, pp. 61-69. 2006.

[13] H. Hiok and B. Qiu. "Fuzzy logic target utilization and prediction for traffic control". Global Telecommunications Conference. Vol. 3, pp. 1644-1648. 2000.

[14] B.A Toledo, V. Muñoz, J. Rogan and C. Tenreiro. "Modeling traffic through a sequence of traffic lights". Physical Review E, Vol. 70, pp. 0161071-0161076. 2004.

[15] R.C. Mayor y J. Cárdenas. "Ingeniería de Tránsito: Fundamentos y aplicaciones". Alfaomega. $7^{\text {a }}$ Edición. México D.F. 2000.

[16] B.D. Greenshield. "A Study in Highway Capacity". Proceedings of the Highway Research Board. Vol. 14, pp. 448-477. 1935.

[17] N.J. Garber and L.A. Hoel. "Traffic and Highway Engineering”. Cengage Learning. $4^{\mathrm{a}}$ Edición. Stanford. USA. 2010.

[18] E. Hurtado y N.L. Torres. "Variables que determinan la localización de cruces peatonales en Bogotá". Tesis de Grado. Departamento de Ingeniería Civil, Universidad Nacional. Bogotá. Colombia. 2000.

[19] Secretaría de Movilidad de Bogotá. "Manual de planeación y diseño para la administración del tránsito y el transporte". Tomo I, Capítulo 5, pp. 50-72. 2005.

[20] T. Takagi and M. Sugeno. "Derivation of fuzzy control rules from human operator's control actions". Proceedings of the IFAC symposium on fuzzy informations, knowledge representation and decision analysis, pp. 55-60. 1983. 
[21] J.S.R. Jang. "ANFIS: adaptive-network-based fuzzy inference system". IEEE Transactions on Systems, Man and Cybernetics. Vol. 23, pp. 665-685. 1993.

[22] S. Zak. "Systems and Control". Oxford University Press. $1^{a}$ Edición. New York. USA. 2003.
[23] J.S.R. Jang. "Input selection for ANFIS learning". Proceedings of the IEEE International Conference on Fuzzy Systems. Vol. 2, pp. 1493-1499. September, 1996. 